\title{
25 Research Square \\ Identification of a potential miRNA-mRNA regulatory network associated with the pathogenesis of cholestatic liver diseases
}

Shuailing Song

West China Hospital of Sichuan University

Xiao Li

West China Hospital of Sichuan University

Chong Geng

West China Hospital of Sichuan University

Yaoyu Guo

West China Hospital of Sichuan University

Chunhui Wang ( $\nabla$ wangch@scu.edu.cn )

West China Hospital of Sichuan University

\section{Research Article}

Keywords: miRNA-mRNA network, cholestatic liver diseases, disease mechanism, SYK, Fc epsilon RI signaling

Posted Date: March 7th, 2022

DOI: https://doi.org/10.21203/rs.3.rs-1400164/v1

License: (c) (i) This work is licensed under a Creative Commons Attribution 4.0 International License. Read Full License 


\section{Abstract \\ Background}

Cholestatic liver diseases (CLDs), including primary sclerosing cholangitis (PSC) and primary biliary cholangitis (PBC), are common digestive diseases that may lead to liver fibrosis, cirrhosis, or even liver failure. However, ursodeoxycholic acid (UDCA), as the first-line drug therapy for PBC, achieves poor results among nearly half of patients. Furthermore, there are few effective ways to cure diseases other than liver transplantation at present. Therefore, it is essential to further explore the disease mechanism in order to find new therapeutic targets.

\section{Methods}

Gene Expression Omnibus (GEO) database (GSE159676) was used to screen differentially expressed hepatic miRNAs between PBC vs. control and PSC vs. control samples, and their interaction was considered as common miRNAs driving CLDs. A miRNA-mRNA network was then constructed based on predicted miRNA-targeted mRNAs. Then enrichment analysis was performed and protein-protein interaction (PPI) networks were constructed. In addition, these DEGs were also validated in models of cholestatic liver injury induced by a -isothiocyanate (ANIT) and bile duct ligation (BDL). Furthermore, the role of hub genes in cholestasis progression, UDCA response, and disease diagnosis were analyzed in GSE166867, GSE79850 and GSE119600, respectively.

\section{Results}

A total of 6 miRNAs and 437 mRNAs were identified as potential mediators in the pathophysiological processes of CLDs. Among them, 8 hub genes were identified (PTPRC, TYROBP, LCP2, RAC2, SYK, TLR2, CD53, and LAPTM5). The functional analysis confirmed that the molecular mechanism was strongly associated with Fc epsilon RI signaling pathway. Combining bioinformatics analysis with animal experimental results, miR-30e and miR-107 were the most significant DE-miRNAs. Additionally, activation of the 8 hub genes was observed in both ANIT-induced cholestasis model and time-course BDL model. Besides, an elevated hepatic SYK level was found in patients with UDCA inefficiency, and SYK in the blood may be an underlying diagnostic indicator for CLDs.

\section{Conclusions}

The present study constructed a miRNA-mRNA network of cholestatic liver diseases, and uncovered that the miR-30e, miR-107 and 8 hub genes could be involved in the pathogenesis of CLDs, providing potential therapeutic targets in CLDs. 


\section{Background}

Cholestatic liver diseases (CLDs) are common digestive diseases characterized by intracellular accumulation of bile acids, self-perpetuation of the inflammatory process, and damage to cholangiocytes and hepatocytes. Primary sclerosing cholangitis (PSC) and primary biliary cholangitis (PBC) are the most common chronic CLDs. If left untreated, cholestasis would lead to fibrosis, cirrhosis, and even liver failure $[1,2]$. However, medications such as ursodeoxycholic acid (UDCA), only delay disease progression. It has been reported that nearly $40 \%$ of PBC patients do not have an adequate biochemical response to UDCA [3]. Until now, liver transplantation has remained the only curative therapy. Thus, in order to identify new therapeutic strategies and drug targets, substantial attention should be paid to further explore the molecular mechanisms involved in the development of CLDs.

microRNAs (miRNAs) are a family of highly conserved small (20-24 nt) endogenous RNAs that do not have the potential to encode proteins but can negatively regulate the expression of target mRNAs by combination with their $3^{\prime}$-untranslated regions (3'-UTRs). Increasing evidence suggests that miRNAs play an important role in hepatic pathology involving inflammation, fibrosis, and cirrhosis [4,5]. Altered miRNA expression in the liver, serum, and peripheral blood mononuclear cells (PBMCs) from PBC or PSC patients has been confirmed by plenty of evidence [6, 7]. Padgett et al [8] reported a distinct miRNA expression signature of liver tissue in patients in the terminal stage of PBC. Afonso et al. [9] suggested aberrant expression of miR-21 in PBC patients and bile-duct ligation (BDL) mice, ablation of which could ameliorate liver necroptosis and improve bile acid homeostasis in BDL mice. However, little research has tried to clarify the miRNA-mRNA regulatory network in CLDs.

In the present research, we examined differentially expressed miRNAs (DE-miRNAs) and mRNAs (DEmRNAs) between cholestatic and normal liver tissue from the GSE159676 dataset. A miRNA-mRNA network was constructed and functional analysis was performed. The expression of the identified DEmiRNAs and hub genes were further verified in cholestasis mouse models. Furthermore, their roles in the disease progression, UDCA response as well as disease diagnosis were explored. Our work may help probe for disease mechanisms that could lead to new therapeutic targets for CLDs.

\section{Materials And Methods}

\section{Data sources}

The microarray datasets were downloaded from the GEO database. The miRNA expression profile dataset GSE159676 was performed on Platform GPL6244, with liver tissues from 12 PSC patients, 3 PBC patients, and 5 healthy people. Differential genes were screened according to the control group and the PSC group or the PBC group. In addition, GSE166867 dataset which contained liver tissues from BDL mice was downloaded. And the gene expression profiles GSE79850 (9 high-risk PBC patients and 7 lowrisk PBC patients) and GSE119600 (45 PSC patients, 90 PBC patients, and 47 healthy controls) were selected for further analysis. A flow chart is presented in Fig. S1. 


\section{Data preprocessing and identification of DE-miRNAs and DE-mRNAs}

GEO2R was used to obtain the differentially expressed genes (DEGs) in the PSC vs. control group and the PBC vs. control group. Because of the small sample size, DE-miRNAs were screened out by an adjusted $\mathrm{P}$ value $<0.05$ and fold change $(F C)>1.2$, and the intersection between the two comparisons was selected as common DE-miRNAs in CLDs. In addition, DE-mRNAs were screened out by an adjusted P-value $<0.05$, and the overlap was further analyzed. To show the expression of DE-miRNAs in different groups, volcano maps and Venn diagrams were drawn by Hiplot (https://hiplot.com.cn), an online bioinformatics data visualization platform.

\section{Prediction of upstream transcription factors of DE-miRNAs}

FunRich (http://www.funrich.org/) [10], a stand-alone software tool mainly for analyzing functional enrichment, was utilized to predict the potential upstream transcription factors of identified DE-miRNAs. $P$ $<0.05$ was considered statistically significant.

\section{Prediction of downstream targeted mRNAs of DE-miRNAs}

The miRWalk V2.0 database was used to predict the targeted mRNAs of DE-miRNAs. Because some gene symbols used in GES159676, such as let-7c, usually refer to two mature miRNAs, 11 corresponding mature miRNAs (hsa-miR-122-5p, hsa-miR-122-3p, hsa-miR-30e-5p, hsa-miR-30e-3p, hsa-let-7c-5p and hsa-let-7c-5p, hsa-miR-107, hsa-miR-503-5p, hsa-miR-503-3p, hsa-miR-192-5p, and hsa-miR-503-3p) were finally chosen for prediction to avoid omitting any relevant candidate miRNA. After that, the predicted mRNAs were further filtered by matching the overlapped DE-mRNAs mentioned above, and then we obtained the miRNA-mRNA pairs according to the negative regulatory relationship between miRNAs and targeted mRNAs. A regulatory network was visualized by Cytoscape software [11].

\section{Gene Ontology (GO) annotation and Kyoto Encyclopedia of Genes and Genomes (KEGG) pathway enrichment analysis of the targeted mRNAs}

ClueGo plugin [12] was used to perform GO and KEGG pathway analysis on mRNAs in the network, which revealed the biological processes (BPs), cellular components (CCs), molecular functions (MFs), and pathways associated with DE-miRNAs. $\mathrm{P}<0.05$ was considered statistically significant.

\section{Protein-protein interaction (PPI) network construction and hub gene identification}

To gain better insight into the relationship among the target genes of the DE-miRNAs, we constructed a PPI network using STRING (http://string-db.org) with confidence scores $\geq 0.4$. The STRING result was further analyzed and visualized by the MCODE plugin and cytoHubba plugin of Cytoscape software. Hub genes, which were defined as genes that played an essential role in the network, were distinguished by crossing the top 2 clustering genes calculated by MCODE algorithm and the top 13 genes calculated by cytoHubba plugin degree method [13]. A miRNA-hub gene regulatory network was then constructed, and a PPI network, as well as KEGG pathway analysis, was further performed. 


\section{Animal model}

Wild-type C57BL/ 6 mice (6-8 weeks, 14-18 g) were purchased from Chengdu Dashuo Biotechnology Co., Ltd. (Sichuan, China). a-Naphthyl isothiocyanate (ANIT) was obtained from Macklin (Shanghai, China) and was used to induce cholestatic liver injury in mice. To be more specific, mice were randomly assigned into the control group and cholestatic liver injury group (ANIT group; for each group, $n=4$ ). The ANIT group was given a single oral dose of ANIT (50 mg/ $\mathrm{kg}$ dissolved in olive oil), and the control group was given a consistent dose of olive oil. All mice were sacrificed 48 hours after treatment. Serum and liver samples were harvested and preserved in liquid nitrogen immediately. All animal study procedures were carried out in line with the National Institutes of Health Guide for the Care and Use of Laboratory Animals.

\section{Sample collection and serum analysis}

Blood samples were collected into centrifuge tubes followed by centrifugation at $3,000 \mathrm{~g}$ for 10 minutes at room temperature to obtain serum. Serum levels of total bilirubin (TBIL), alkaline phosphatase (ALP), alanine aminotransferase (ALT), and aspartate aminotransferase (AST) were determined using standard clinical chemistry techniques.

\section{RNA isolation and RT-qPCR analysis}

Liver tissues were collected, and total RNA from the liver samples was extracted using a Total RNA kit (Forgene, Sichuan, China) according to the manufacturer's instructions. A miRNA reverse transcription kit

(Vazyme, Jiangsu, China) and a first-strand cDNA synthesis kit (Forgene, Sichuan, China) were applied to synthesize cDNA. RT-qPCR was accomplished using SYBR Green PCR Master Mix (Forgene, Sichuan, China). Primers for reverse transcription and RT-qPCR are presented in Table S1. The same reverse primer with the sequence 5'-AGTGCAGGGTCCGAGGTATT-3' was used for all miRNAs. The average expression levels of liver miRNAs and mRNAs were normalized to U6 and GAPDH, respectively. Relative gene expression was determined by the $2^{-\Delta \Delta C t}$ method.

\section{Statistical analysis}

All data are presented as the mean \pm standard error (SEM). Student's t-test was carried out to compare two groups, and one-way analysis of variance (ANOVA) was applied for multigroup $(\geq 3)$ comparisons by SPSS 16.0 (Chicago, USA). The bar charts and the ROC curve analysis were completed by GraphPad Prism 8.0 (California, USA). $\mathrm{P}<0.05$ was considered statistically significant.

\section{Results}

\section{Identification of DE-miRNAs}

According to the filtering criterion described before, the cutoff value for FC of DE-miRNAs was 1.2. We identified 8 downregulated DE-miRNAs between the control group and the PBC group. When comparing the PSC group to the control, we found 11 altered miRNAs, including 1 upregulated miRNA and 10 
downregulated miRNAs. The distributions of DE-miRNA expression between the control group and the PBC or PSC group were intuitively illustrated by volcano maps (Fig. 1a and 1b). Furthermore, the intersection of the candidate DE-miRNAs from the two comparisons was analyzed and visualized by a Venn diagram (Fig. 1C). The shared DE-miRNAs are also listed in Table 1, including miR-122, miR-30e, let7c, miR-107, miR-503, and miR-192.

\section{Prediction of transcription factors and the target genes of the DE-miRNAs}

It is suggested that miRNAs could be affected by transcription factors (TFs) and negatively regulate the expression of targeted genes. Herein, to understand the underlying regulatory network of the identified DEmiRNAs, we first utilized FunRich software to predict the TFs of the 6 DE-miRNAs. The top 10 significant TFs are shown in Fig. 2a, among which EGR1 has the greatest difference and SP1 regulates the most miRNAs (57\%).

Additionally, a total of 437 upregulated genes were mapped into 11 downregulated DE-miRNAs. A miRNAmRNA network was visualized in Cytoscape (Fig. 2b). Information on the targeted genes is also shown in Table 2.

\section{Enrichment analysis of the target mRNAs}

To investigate the functions of the DEGs, GO annotation and KEGG pathway analysis were performed utilizing the Cluego plugin of Cytoscape. The results showed that BP terms were significantly enriched in lymphocyte differentiation and activation and immune system-related function (Fig. 3a). The CC terms included side of membrane, secretory granule membrane, anchoring junction, actin cytoskeleton, and MHC class $₫$ protein complex (Fig. 3b). And the most enriched entries for MF terms were activities associated with GTPase, G protein-coupled purinergic nucleotide receptor, protein tyrosine phosphatase, and immune receptor (Fig. 3c). In addition, the most important pathways of KEGG are shown in Fig. 3d, including those related to inflammation, the immune system, and various infections, which are critical for the pathological development of CLDs.

\section{Construction of the PPI network and identification of hub genes}

To distinguish the connections among the 437 target genes, we mapped the PPIs using the logical data originating from the STRING database. 437 nodes and 1442 edges were obtained with an average local clustering coefficient of 0.399 . We further used the MCODE plugin to process the network data to identify gene clusters, and the clusters with the top 5 highest scores are shown in Fig. $4 a$. We also used CytoHubba plugin to rank the nodes by the degree method (Fig. 4b). The top 13 genes are listed in Table 3. Next, to obtain the hub genes, genes in Cluster 1 and Cluster 2 were further filtered by matching the top 13 genes with the highest degree scores (Fig. 4c). Then, we got 8 hub genes: PTPRC, TYROBP, LCP2, RAC2, SYK, TLR2, CD53, and LAPTM5. A PPI network and a miRNA-hub gene regulatory network were also constructed (Fig. 4d-e). Notably, KEGG pathway analysis revealed that the Fc epsilon RI signaling 
pathway, which was involved with LCP2, RAC2 and SYK, was significantly enriched, suggesting this signaling pathway was important in the development of CLDs (Fig. 4f).

\section{Verification of the identified miRNAs and mRNAs in ANIT-induced cholestasis mice}

ANIT, a well-characterized hepatotoxic agent that causes cholestasis by injuring bile duct epithelial cells and hepatocytes, is widely used to emulate human acute intrahepatic cholestasis [14]. To verify the results of the bioinformatics analysis, we then detected the levels of the identified miRNAs in ANITinduced cholestatic liver injury mice. As shown in Fig. 5a, we observed an enlarged gall bladder and dark bile inside in the ANIT-treated mice, which suggested that the cholestasis model had been established successfully. In addition, serum biochemical detection also confirmed this result (Fig. S2). As expected, the expression of DE-miRNAs was significantly decreased in the ANIT group compared with the control group (Fig. 5b), except miR-503-5p, which did not reach a significant difference despite a downregulation trend in the ANIT group.

After the verification of the DE-miRNAs, we continued to analyze the expression of the hub genes in the ANIT-treated mice. Consistently, ANIT-induced cholestasis significantly stimulated the liver expression of the hub genes (Fig. 5c). Taken together, these findings implied that the miRNA-mRNA regulatory network (except miR-503-5p) may participate in the ANIT-induced cholestatic liver injury.

\section{Verification of the identified miRNAs and mRNAs in BDL-induced cholestasis mice}

The BDL mouse model is a classic animal model to induce extrahepatic cholestasis. Therefore, we utilized gene expression data from GSE166867 to verify the level of the identified DE-miRNAs between BDL mice and sham operation mice. As shown in Fig. 6a, 5 forementioned DE-miRNAs except miR-503 were detected in this dataset. The expression of miR-30e and miR-107 was significantly decreased 21 days after BDL ( $21 \mathrm{~d} B D L)$ compared to the sham group, which was consistent with our previous results. In contrast, the expression of the other 3 miRNAs remained constant between the two groups, indicating that they were not involved in the BDL-induced cholestatic liver injury.

We next analyzed the alteration of the hub genes. As shown in Fig.6b-i, compared to the sham group, the 8 hub genes were remarkably increased in the $21 \mathrm{~d}$ BDL group, which was consistent with our previous results. Changes could occur in the gene signature during disease progression. To better master the role of the DEGs in the dynamic development of cholestatic liver injury, we analyzed the changes of hub genes early after BDL. Results showed that BDL dramatically enhanced most hub genes levels both 3 days and 7 days after BDL ( $3 \mathrm{~d} B D L$ and $7 \mathrm{~d} B D L$ ) compared to the sham group. Notably, among the 8 hub genes, except TLR2, the $7 \mathrm{~d}$ and $21 \mathrm{~d}$ BDL groups exhibited a significant upregulation trend compared to the $3 \mathrm{~d}$ BDL group. Although this trend weakened from 7 days to 21 days after BDL, we still found that most genes continued to increase in the process of cholestatic liver injury. These findings suggested that these hub genes were continuously activated throughout the process of cholestasis, playing an important role in the cholestasis-mediated liver injury. 


\section{Analysis of the DE-mRNAs and UDCA response}

Recently, UDCA dominates the drug therapy of cholestasis. However, nearly half of PBC patients face the dilemma that they do not fully respond to UDCA. Thus, we were curious about whether these identified DE-mRNAs were associated with UDCA response.

Dataset GSE79850, which contained the first liver biopsies from 9 PBC patients requiring liver transplantation (high-risk group) and 7 PBC patients whose condition was greatly controlled by UDCA therapy (low-risk group), was chosen to compare levels of the identified hub genes between the two groups (Fig. 7a-d). Interestingly, we found that the level of SYK in the high-risk group was more than twice as high as that in the low-risk group $(79.59 \pm 11.48$ vs $31.18 \pm 3.55, P=0.003)$, which implied that SYK may play an important role in the response to UDCA.

\section{Analysis of the DEGs in the blood of patients with CLDs}

According to the result of functional analysis, DEGs were enriched in the pathway associated with hematopoietic cells, which prompted us to speculate whether levels of the DE-miRNAs and DE-mRNAs in peripheral blood of patients with CLDs were changed. The GSE119600 dataset was selected for further analysis. As a result, 5 hub genes (PTPRC, RAC2, SYK, TLR2, and LAPTM5) were detected in the blood samples (Fig. 8a-e). Most hub genes remarkably increased in the PSC or PBC group when compared to the control group, except PTPRC, which remained constant among the 3 groups. After that, to investigate the efficacy of these altered genes as potential biomarkers of CLDs, we performed ROC curve analysis. As shown in Fig. $8 \mathrm{f}-\mathrm{i}$, the AUC values of the 4 DE-mRNAs were all greater than 0.6 when used to diagnose both PSC and PBC, especially SYK and LAPTM5, which were greater than 0.7 . These results indicated that SYK and LAPTM5 in the blood had potential as diagnostic markers in CLDs.

\section{Discussion}

MiRNAs represent a new area of interest in the investigation of CLDs. In the present study, we used bioinformatic analysis to identify cholestasis-related genes based on the published dataset obtained from GEO. We found the intersection of PSC vs. control comparison and PBC vs. control comparison to determine the most valuable miRNAs. By constructing a miRNA-mRNA network, a total of 6 miRNAs and 437 mRNAs were identified as potential mediators in the pathophysiological process of CLDs.

However, PBC and PSC are typical autoimmune diseases, while CLDs were characterized by accumulation of bile acid including its toxic effect on cholangiocytes and hepatocytes. In order to figure out whether the DEGs participate in the cholestasis process and liver injury, we then verified their expression in ANIT-induced intrahepatic cholestasis as well as BDL-induced extrahepatic cholestasis model. As a result, miR-30e and miR-107 were significantly downregulated in both cholestasis models, suggesting the two miRNAs may be closely related to cholestasis. In fact, some evidence has suggested the role of miR-30e and miR-107 in hepatopathology. Overexpression of miR-30e decreased the lipid synthesis-related proteins SREBP-1c and FASN in hepatocytes. It could also inhibit autophagy activation 
in HCV-infected hepatocytes [15]. In addition, it was also found that miR-30e could take part in HBVinduced liver fibrosis progression by targeting P4HA2 [16]. MiR-107 is closely associated with metabolic pathways [17, 18], and a high-fat diet could alter its hepatic expression [19]. In turn, miR-107 could inhibit fatty acid synthase activation in human liver HepG2 cells [20]. Wang et al. [21] demonstrated that miR107 could participate in the activation of hepatic stellate cells induced by exosomes derived from palmitic acid-treated hepatocytes. Our study first reported a possible role of miR-30e and miR-107 in the CLDs, providing a novel insight into miRNAs in the CLDs.

To determine whether any of these DE-miRNAs could lead to a better understanding of the underlying molecular mechanism that perpetuates cholestasis, we searched for targeted genes, and 8 genes were determined to be the key genes in the miRNA-mRNA network. KEGG analysis were enriched in the Fc epsilon RI signaling pathway, which plays an important role in the activation of mast cells (MCs). In fact, the relationship between MCs and cholestasis has been widely reported in the literature. It was suggested that MCs migrate into the liver and are activated in patients with cholestasis. Aller et al. [22] found that an increase in splanchnic mast cells aggravated the inflammatory response in cholestasis rats. In turn, inhibition of MC mediators decreases ductular reaction and liver fibrosis [23]. However, limited information is available regarding the regulation of MCs in the CLDs.

In the recent work, the expression of the 8 hub genes was validated in both intrahepatic and extrahepatic cholestasis mouse models. Notably, Fc Epsilon RI signaling pathway was significantly enriched. This provided reasonable speculation that this pathway was involved in cholestasis, contributing to the underlying mechanism of MCs regulation in CDLs pathology.

Besides, we were surprised to find an elevated expression of SYK in patients with an insufficient UDCA response. On one hand, SYK could combine Fc epsilon RI (IgE receptor) to initiate MC degranulation. On the other hand, UDCA could attenuate biliary injury, inflammation, and fibrosis via suppressing MC activation [24]. The antagonistic effect on MCs may be a partial interpretation for patients without an adequate response to UDCA, and it could also be an underlying therapeutic target.

There are several limitations to our present study. First, because of the relatively small sample size obtained from GSE159676, we may generate bias when performing data analysis. Therefore, more samples should be included for further research. Second, more mechanistic studies are needed to clarify the regulatory relationship between the DE-miRNAs and possible target genes.

\section{Conclusions}

The present study constructed a miRNA-mRNA network of CLDs, and 6 DE-miRNAs and 8 hub genes were viewed as potential mediators in disease development. Combining bioinformatics analysis and animal experiment studies, miR-30e and miR-107 were the most significantly altered miRNAs. And they may participate in the pathogenesis of CLD by regulating Fc Epsilon RI signaling pathway through LCP2/RAC2/SYK. Our findings provided a deeper understanding of CLDs as well as potential therapeutic targets. 


\section{Abbreviations}

ANIT

a-Naphthyl isothiocyanate

ALP

alkaline phosphatase

BP

biological process

CC

cellular component

CLDs

cholestatic liver diseases

DEGs

differentially expressed genes

DE-miRNA

differentially expressed microRNA

DE-mRNAs

differentially expressed mRNA

GEO

Gene Expression Omnibus

GO

Gene Ontology

MF

molecular function

KEGG

Kyoto Encyclopedia of Genes and Genomes

PBC

primary biliary cholangitis

PPI

protein-protein interaction

PSC

Primary sclerosing cholangitis

TBIL

total bilirubin

UDCA

ursodeoxycholic acid.

\section{Declarations}

Ethics approval and consent to participate 
The present study was approved by the Ethical Committee of the West China Hospital of Sichuan University (Ethical Committee Number: 20220121002). All animal experiments were conducted in accordance with the guidelines of the Ethical Committee of the West China Hospital of Sichuan University. Additionally, the animal experiments also adhered to the ARRIVE guidelines (https://arriveguidelines.org).

\section{Consent for publication}

Not applicable.

\section{Availability of data and materials}

The datasets generated and/or analysed during the current study are available in the GEO database (https://www.ncbi.nlm.nih.gov/geo/).

\section{Competing interests}

The authors declare there are no competing interests.

\section{Funding}

The work was supported by National Natural Science Foundation of China (82070544) and Sichuan Science and Technology Program (2020YFS0239).

\section{Authors' Contributions}

X.L. and C.W. conceived and designed the research; S.S., X.L., C.G., and Y.G. performed experiments; S.S., X.L. and C.G. analyzed data; S.S. and X.L. prepared figures and drafted manuscript; C.W. edited and revised the manuscript; all authors approved the final version of the manuscript.

\section{Acknowledgements}

Not applicable.

\section{Authors' information}

${ }^{1}$ Department of Gastroenterology, West China Hospital of Sichuan University, Chengdu 610041, China;

2 Division of Digestive Diseases, West China Hospital of Sichuan University, Chengdu 610041, China

\section{References}

1. Santiago P, Scheinberg AR, Levy C. Cholestatic liver diseases: new targets, new therapies. Therap Adv Gastroenterol 2018;11:1756284818787400. 
2. Woolbright BL, Jaeschke H. Inflammation and Cell Death During Cholestasis: The Evolving Role of Bile Acids. Gene Expr 2019;19:215-228.

3. Pares A, Caballeria $L$, Rodes J. Excellent long-term survival in patients with primary biliary cirrhosis and biochemical response to ursodeoxycholic Acid. Gastroenterology 2006;130:715-20.

4. Szabo G, Bala S. MicroRNAs in liver disease. Nat Rev Gastroenterol Hepatol 2013;10:542-52.

5. Zhao Z, Lin CY, Cheng K. siRNA- and miRNA-based therapeutics for liver fibrosis. Transl Res 2019;214:17-29.

6. Marin JJ, Bujanda L, Banales JM. MicroRNAs and cholestatic liver diseases. Curr Opin Gastroenterol 2014;30:303-9.

7. Huang $C$, Xing $X$, Xiang $X$, et al. MicroRNAs in autoimmune liver diseases: from diagnosis to potential therapeutic targets. Biomed Pharmacother 2020;130:110558.

8. Padgett KA, Lan RY, Leung PC, et al. Primary biliary cirrhosis is associated with altered hepatic microRNA expression. J Autoimmun 2009;32:246-53.

9. Afonso MB, Rodrigues PM, Simao AL, et al. miRNA-21 ablation protects against liver injury and necroptosis in cholestasis. Cell Death Differ 2018;25:857-872.

10. Pathan M, Keerthikumar S, Ang CS, et al. FunRich: An open access standalone functional enrichment and interaction network analysis tool. Proteomics 2015;15:2597-601.

11. Smoot ME, Ono K, Ruscheinski J, et al. Cytoscape 2.8: new features for data integration and network visualization. Bioinformatics 2011;27:431-2.

12. Bindea G, Mlecnik B, Hackl H, et al. ClueGO: a Cytoscape plug-in to decipher functionally grouped gene ontology and pathway annotation networks. Bioinformatics 2009;25:1091-3.

13. Chin $\mathrm{CH}$, Chen $\mathrm{SH}, \mathrm{Wu} \mathrm{HH}$, et al. cytoHubba: identifying hub objects and sub-networks from complex interactome. BMC Syst Biol 2014;8 Suppl 4:S11.

14. Mariotti V, Strazzabosco M, Fabris L, et al. Animal models of biliary injury and altered bile acid metabolism. Biochim Biophys Acta Mol Basis Dis 2018;1864:1254-1261.

15. Sasaki R, Sur S, Cheng Q, et al. Repression of MicroRNA-30e by Hepatitis C Virus Enhances Fatty Acid Synthesis. Hepatol Commun 2019;3:943-953.

16. Feng GX, Li J, Yang Z, et al. Hepatitis B virus X protein promotes the development of liver fibrosis and hepatoma through downregulation of miR-30e targeting P4HA2 mRNA. Oncogene 2017;36:68956905.

17. Trajkovs.ki M, Hausser J, Soutschek J, et al. MicroRNAs 103 and 107 regulate insulin sensitivity. Nature 2011;474:649-53.

18. Wilfred BR, Wang WX, Nelson PT. Energizing miRNA research: a review of the role of miRNAs in lipid metabolism, with a prediction that miR-103/107 regulates human metabolic pathways. Mol Genet Metab 2007;91:209-17.

19. Joven J, Espinel E, Rull A, et al. Plant-derived polyphenols regulate expression of miRNA paralogs miR-103/107 and miR-122 and prevent diet-induced fatty liver disease in hyperlipidemic mice. 
Biochim Biophys Acta 2012;1820:894-9.

20. Sun D, Zhao T, Long K, et al. Triclosan down-regulates fatty acid synthase through microRNAs in HepG2 cells. Eur J Pharmacol 2021;907:174261.

21. Wang W, Li F, Lai X, et al. Exosomes secreted by palmitic acid-treated hepatocytes promote LX-2 cell activation by transferring miRNA-107. Cell Death Discov 2021;7:174.

22. Aller MA, Martinez V, Arias A, et al. Mast cell-mediated splanchnic cholestatic inflammation. Clin Res Hepatol Gastroenterol 2019;43:561-574.

23. Kyritsi K, Kennedy L, Meadows V, et al. Mast Cells Induce Ductular Reaction Mimicking Liver Injury in Mice Through Mast Cell-Derived Transforming Growth Factor Beta 1 Signaling. Hepatology 2021;73:2397-2410.

24. Meng F, Kennedy L, Hargrove L, et al. Ursodeoxycholate inhibits mast cell activation and reverses biliary injury and fibrosis in Mdr2(-/-) mice and human primary sclerosing cholangitis. Lab Invest 2018;98:1465-1477.

\section{Tables}

Table 1 The DE-miRNAs

\begin{tabular}{|llllll|}
\hline \multirow{2}{*}{ Symbol } & \multicolumn{2}{l}{ PSC vs. Ctrl } & \multicolumn{2}{l}{ PBC vs. Ctrl } & \multirow{2}{*}{ Up/Down } \\
\cline { 2 - 5 } & LogFC & Adj.P & LogFC & Adj.P & \\
\hline hsa-miR-122 & -1.97 & $3.58 \mathrm{E}-08$ & -1.84 & $4.73 \mathrm{E}-05$ & Down \\
\hline hsa-miR-30e & -0.69 & $2.72 \mathrm{E}-04$ & -0.75 & $5.85 \mathrm{E}-03$ & Down \\
\hline hsa-let-7c & -0.75 & $5.91 \mathrm{E}-04$ & -0.76 & $1.06 \mathrm{E}-02$ & Down \\
\hline hsa-miR-107 & -0.31 & $8.44 \mathrm{E}-04$ & -0.38 & $1.72 \mathrm{E}-02$ & Down \\
\hline hsa-miR-503 & -0.28 & $3.70 \mathrm{E}-04$ & -0.26 & $1.64 \mathrm{E}-02$ & Down \\
\hline hsa-miR-192 & -0.27 & $1.44 \mathrm{E}-03$ & -0.34 & $3.50 \mathrm{E}-02$ & Down \\
\hline
\end{tabular}

Table 2 The target number of the DE-miRNAs 


\begin{tabular}{|ll|}
\hline miRNAs & Target-gene count \\
\hline hsa-miR-122-5p & 326 \\
\hline hsa-miR-122-3p & 123 \\
\hline hsa-miR-30e-5p & 134 \\
\hline hsa-miR-30e-3p & 215 \\
\hline hsa-let-7c-5p & 356 \\
\hline hsa-let-7c-3p & 217 \\
\hline hsa-miR-107 & 373 \\
\hline hsa-miR-503-5p & 367 \\
\hline hsa-miR-503-3p & 388 \\
\hline hsa-miR-192-5p & 155 \\
\hline hsa-miR-192-3p & 312 \\
\hline
\end{tabular}

Table 3 Top 13 genes in network ranked by degree method 


\begin{tabular}{lll} 
Rank & Symbol & Score \\
\hline 1 & PTPRC & 84 \\
\hline 2 & TYROBP & 62 \\
\hline 3 & LCP2 & 51 \\
\hline 4 & RAC2 & 45 \\
\hline 5 & CD44 & 40 \\
\hline 6 & CD48 & 40 \\
\hline 7 & SYK & 39 \\
\hline 8 & FOXP3 & 38 \\
\hline 9 & TLR2 & 38 \\
\hline 10 & IL7R & 37 \\
\hline 11 & CXCL8 & 37 \\
\hline 12 & CD53 & 37 \\
\hline 13 & LAPTM5 & 37
\end{tabular}

\section{Figures}

Fig1

a

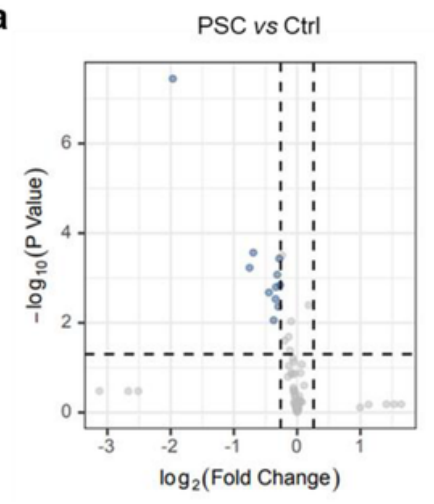

b

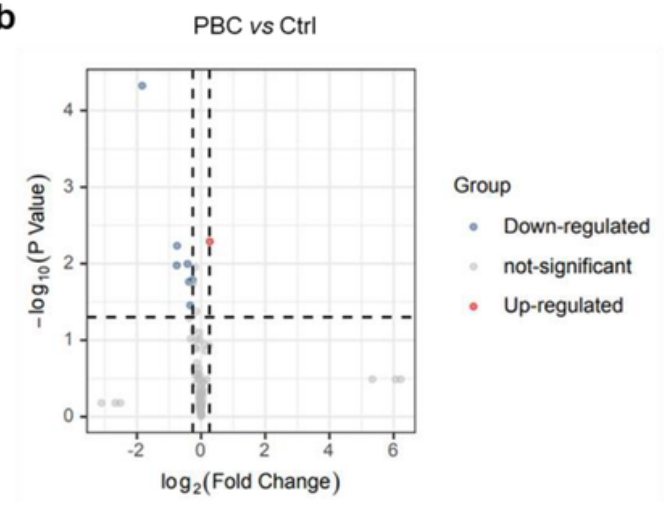

c

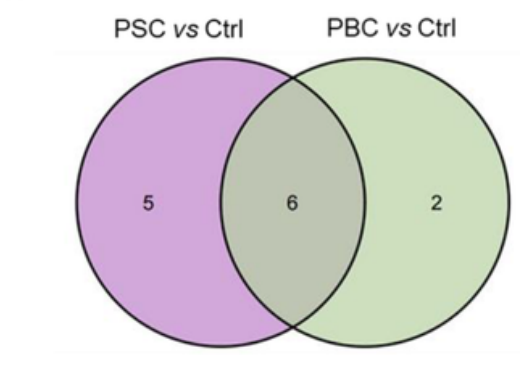

Figure 1

Group

- Down-regulated not-significant

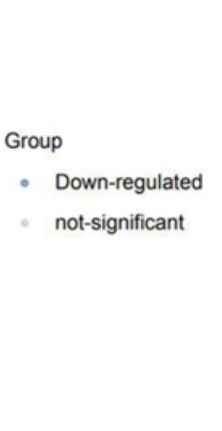


Identification of differentially expressed miRNAs. Volcano plot of differentially expressed miRNAs of PSC vs Control (a) and PBC vs Control (b) in GSE159676; (c) A Venn diagram between PSC vs Ctrl comparison and PBC vs Ctrl comparison. The overlapping part represents the differentially expressed miRNAs shared by the two comparisons, accounting for a total of 6 .

Fig2

a

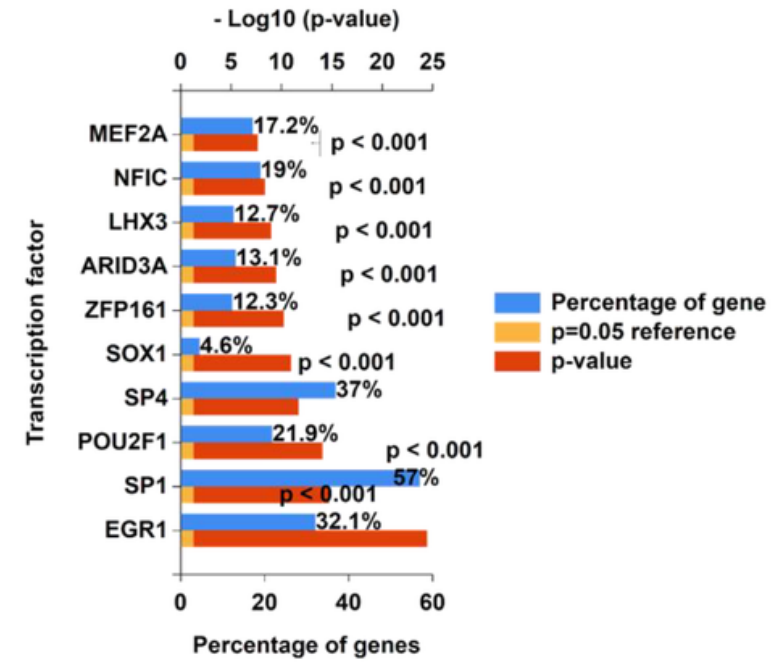

b

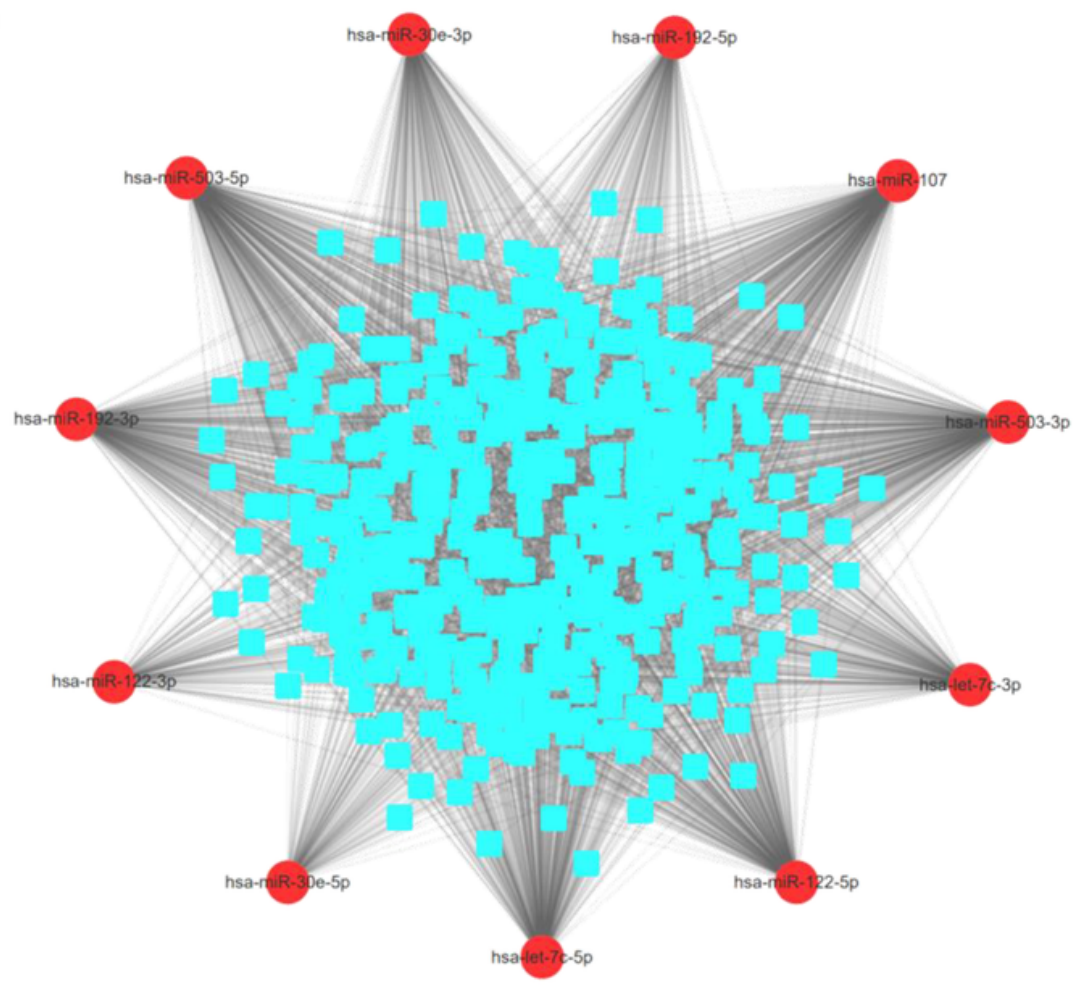

Figure 2

Upstream transcription factors and downstream target genes of DE-miRNAs. (a) Transcription factors predicted by FunRich; (b) The miRNA-mRNA network. The red circles represent miRNAs, and the blue circles represent target genes. 
Fig3
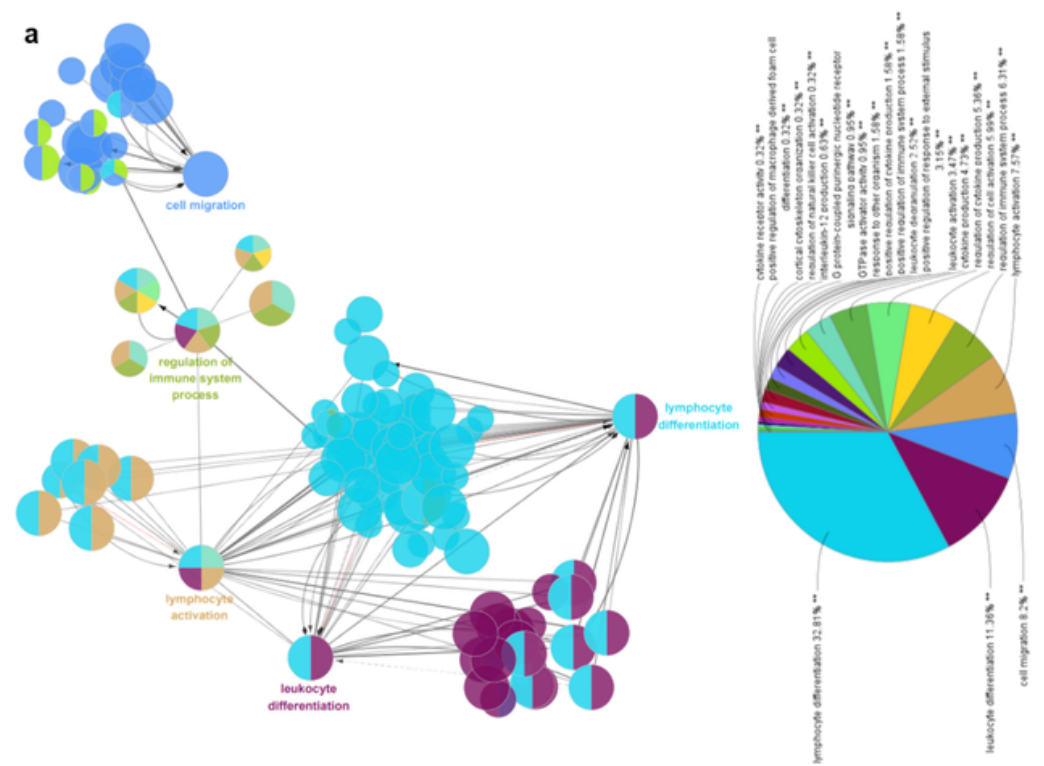

b
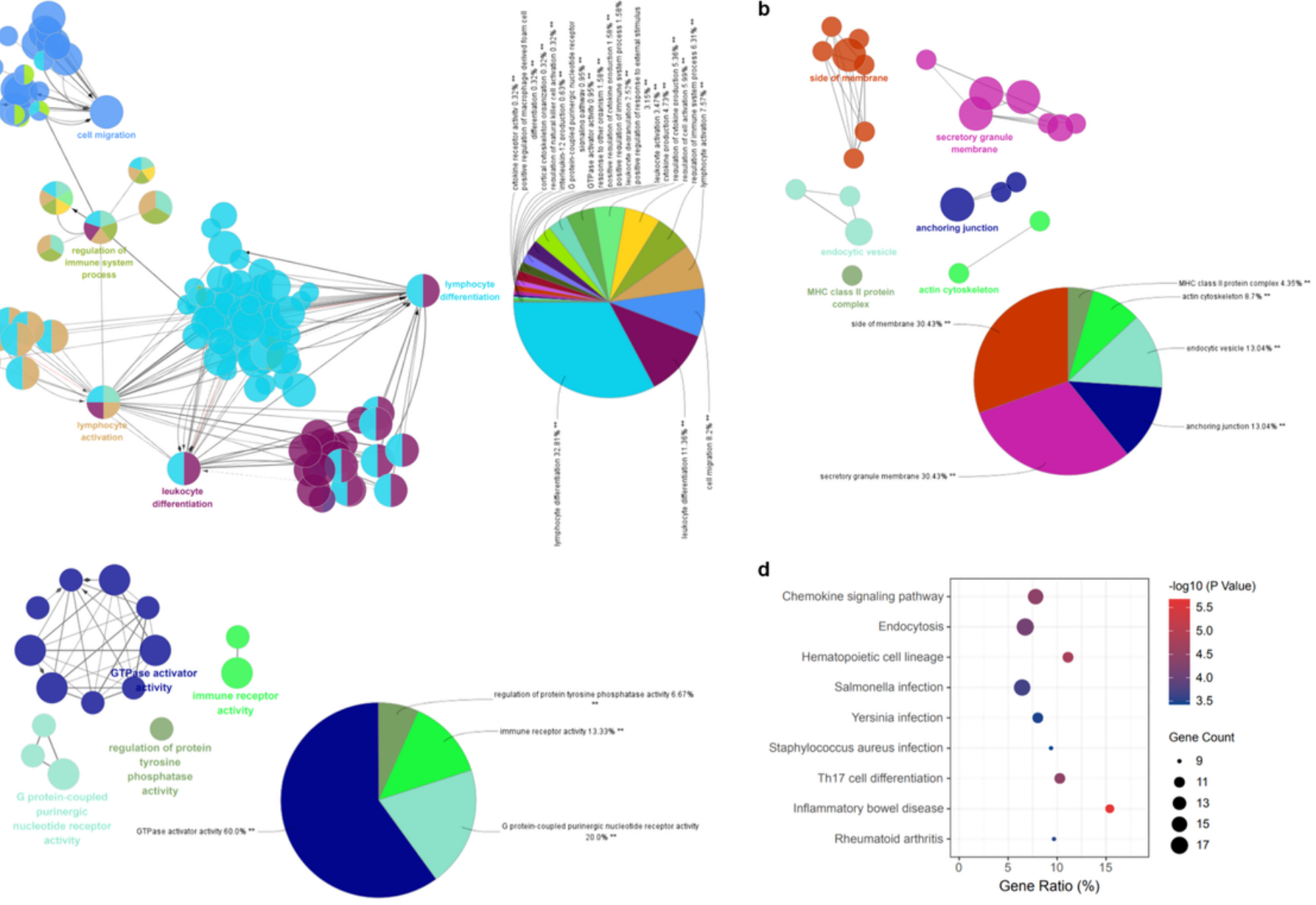

d

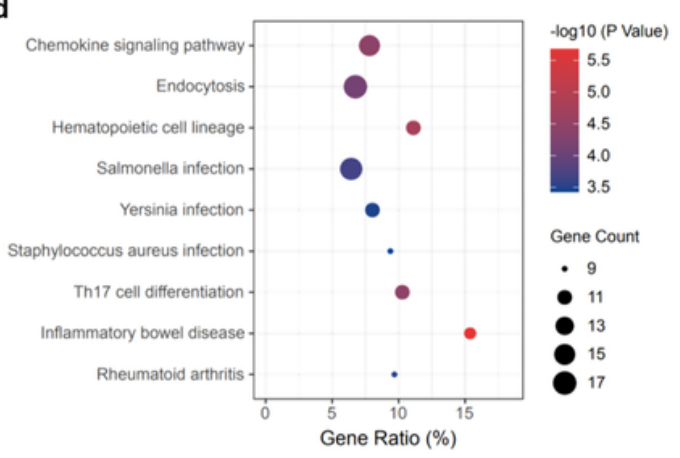

Figure 3

GO and KEGG terms of DE-mRNAs in cholestasis liver diseases. (a) Biological process; (b) Cellular component; (c) Molecular function; (d) KEGG pathway enrichment. 
Fig4

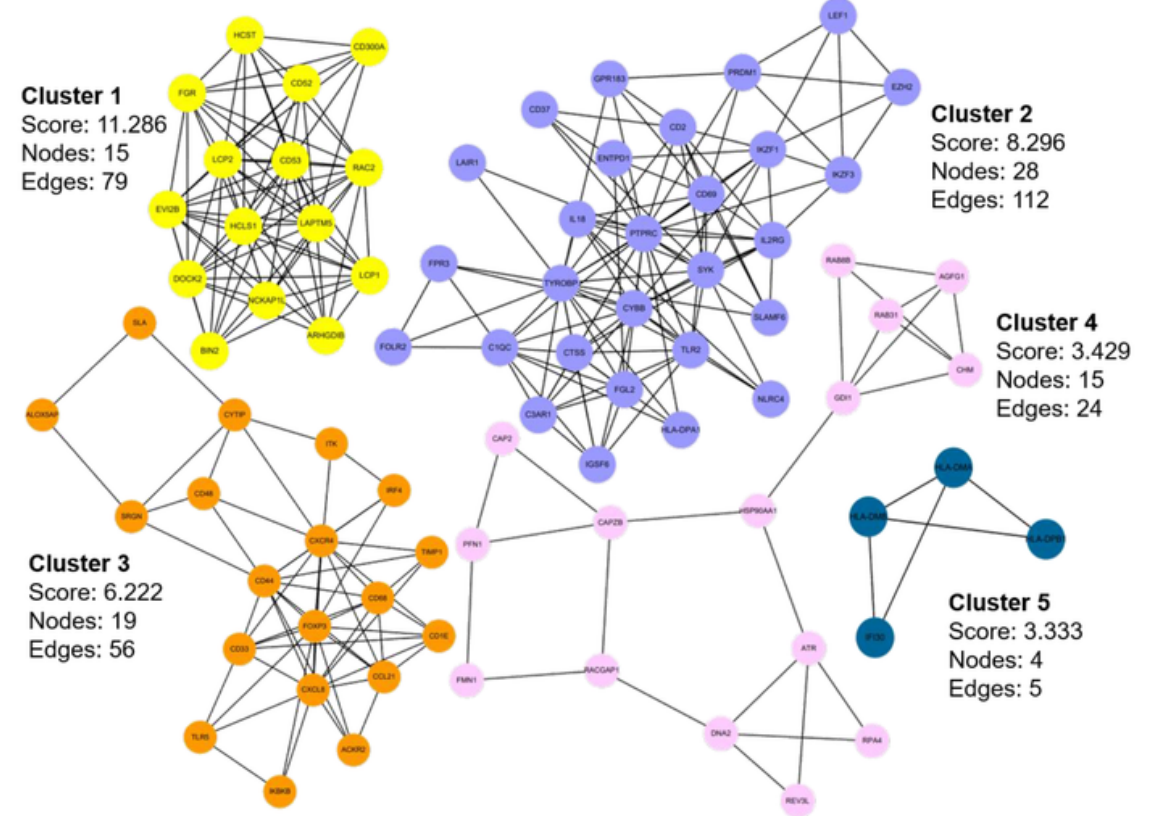

b

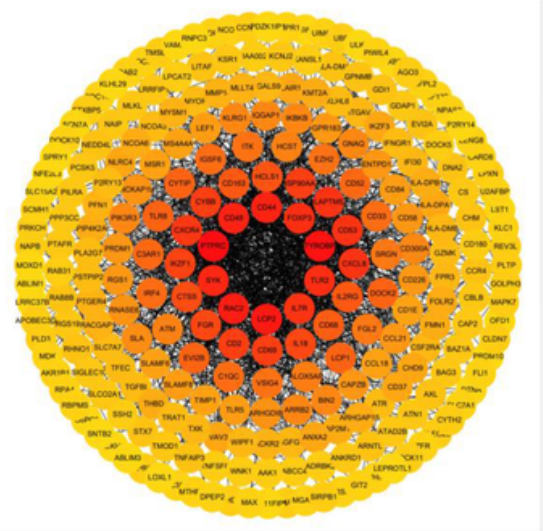

Genes of cluster $1 \& 2$

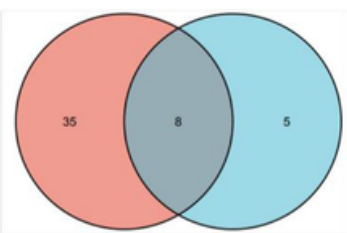

Top 13 genes ranked by degree

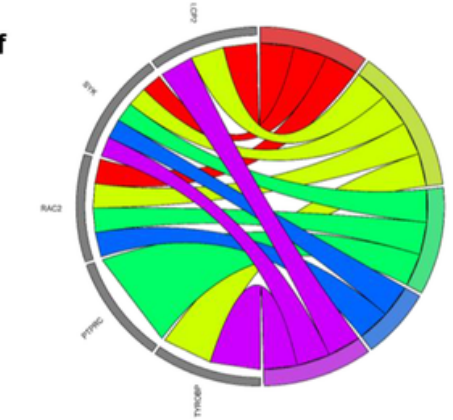

Fc epsilon RI signaling pathway

Natural killer cell mediated cytotoxicity

Fc gamma R-mediated phagocytosis

B cell receptor signaling pathway

Osteoclast differentiation

\section{Figure 4}

Identification of the hub genes in the PPI network. (a) Top 5 clusters identified by MCODE; (b) Genes ranked by the degree method by cytoHubba; genes are labeled with different colors according to their degree scores, and those with the highest scores are marked with dark red. (c) A Venn diagram of genes from the top 2 clusters and top 13 degree scores; (d) PPI network of hub genes; (e) miRNA-hub gene network; (f) KEGG pathway enrichment. 
Fig5
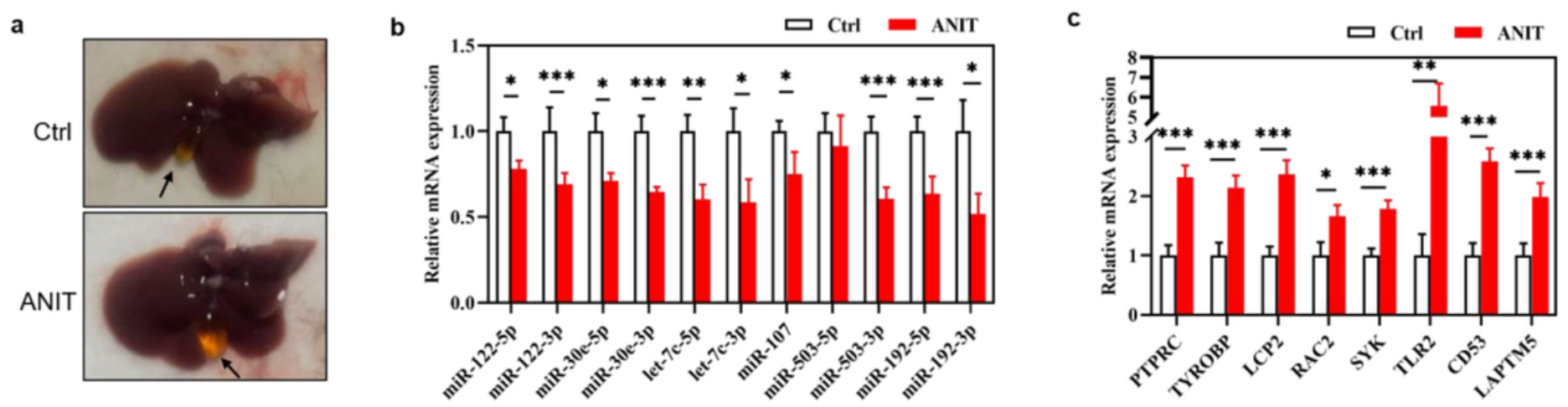

Figure 5

Verification of DEGs in ANIT-induced cholestatic liver injury mice. (a) Liver specimens from ANIT-treated and control mice. (b) Hepatic DE-miRNA expression; (c) Hepatic DE-mRNA expression. ${ }^{*} \mathrm{P}<0.05, * * \mathrm{P}<$ $0.01,{ }^{*} * * \mathrm{P}<0.001$.

Fig6

a
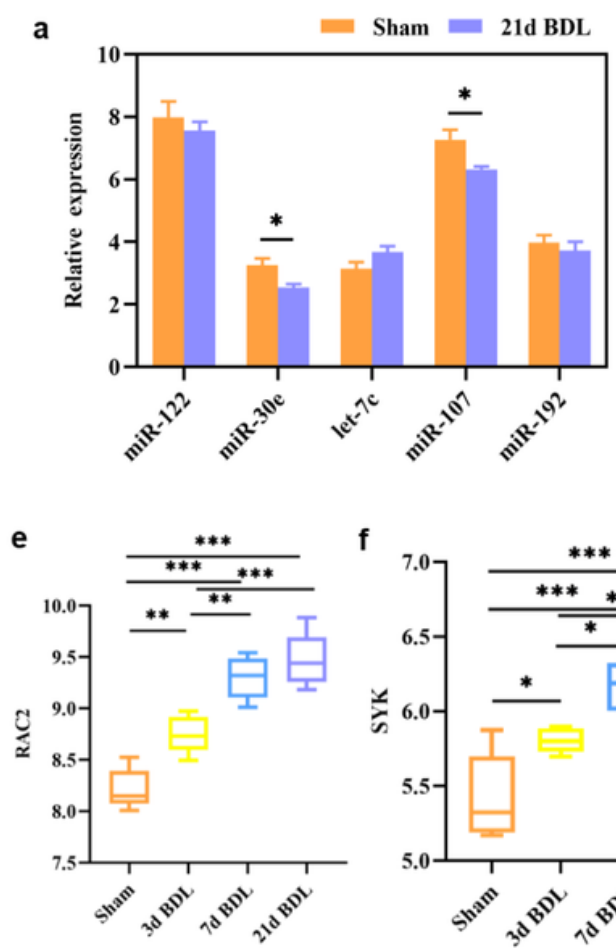
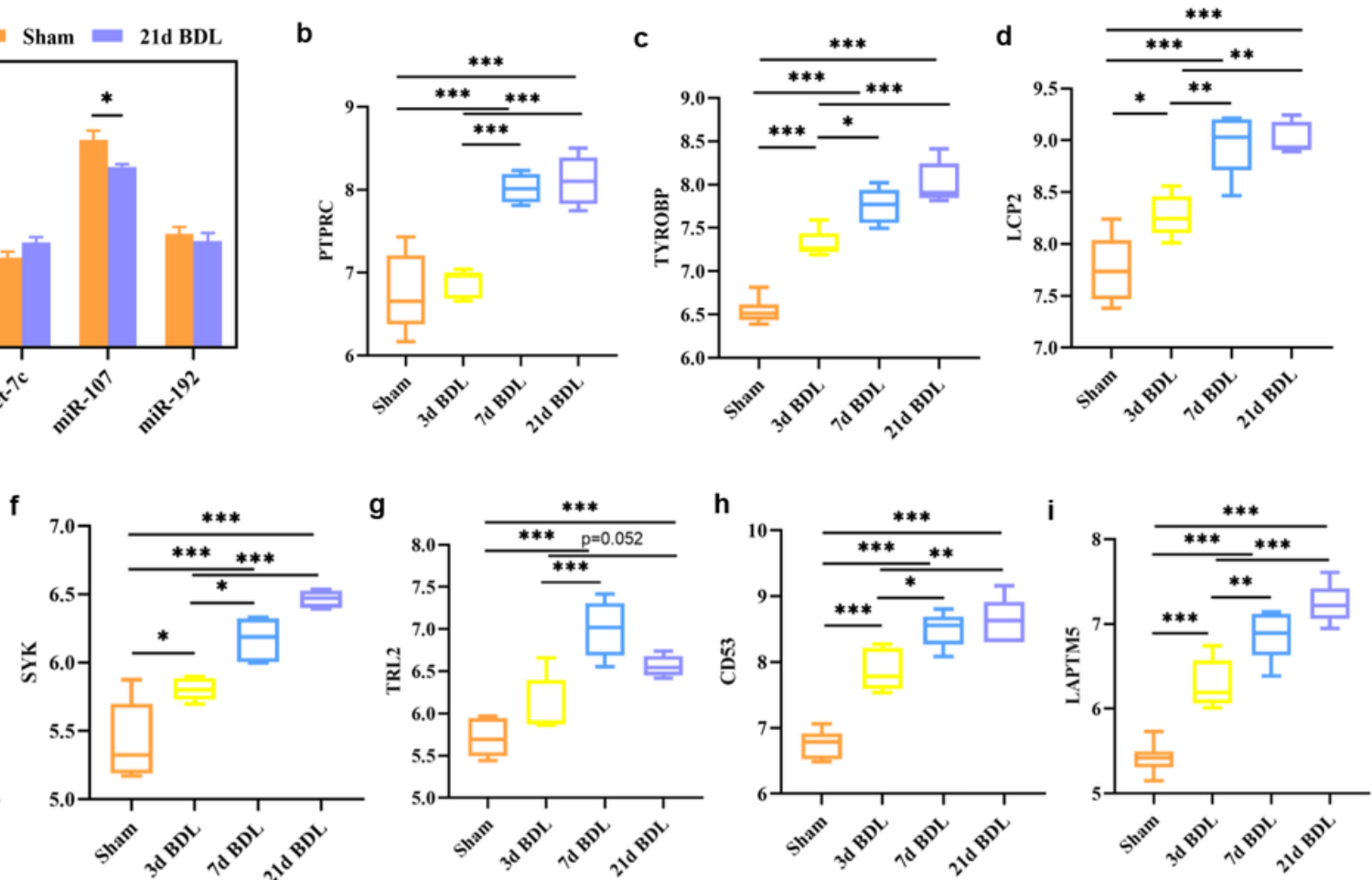

Figure 6 
Verification of DEGs in BDL-induced cholestatic liver injury mice in GSE 166867. (a) Hepatic DE-miRNA expression; (b-i) Expression of the hub genes in the liver of BDL mouse models at different time points. *P $<0.05, * * \mathrm{P}<0.01, * \star * \mathrm{P}<0.001$.

Fig7

a

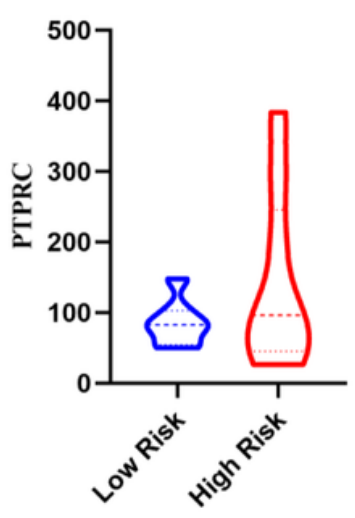

b

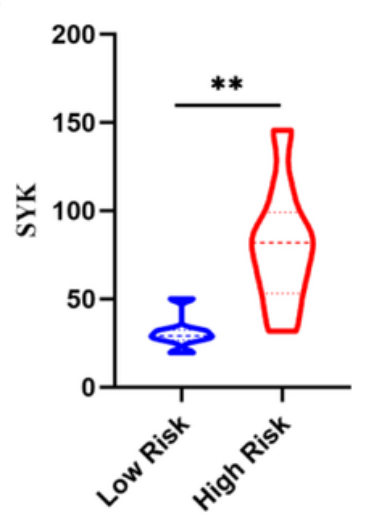

C

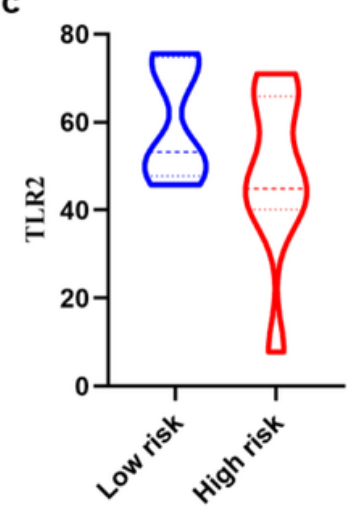

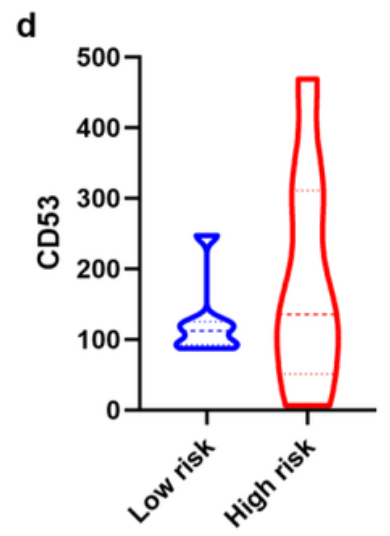

Figure 7

Expression of hub genes in high-risk PBC patients in GSE79850. Expression of hepatic PTPRC (a), SYK (b), TLR2 (c) and CD53 (d) in PBC patients with low risk (who completely responded to UDCA) or high risk (who needed liver transplantation). ${ }^{\star *} \mathrm{P}<0.01$. 
Fig8

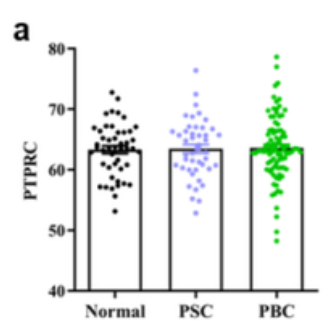

b

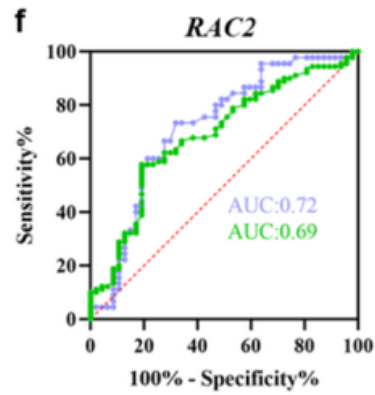

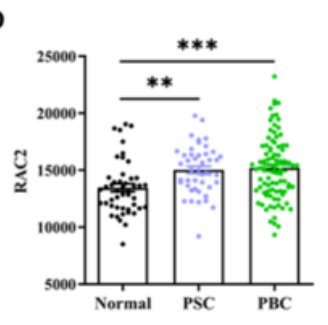

C
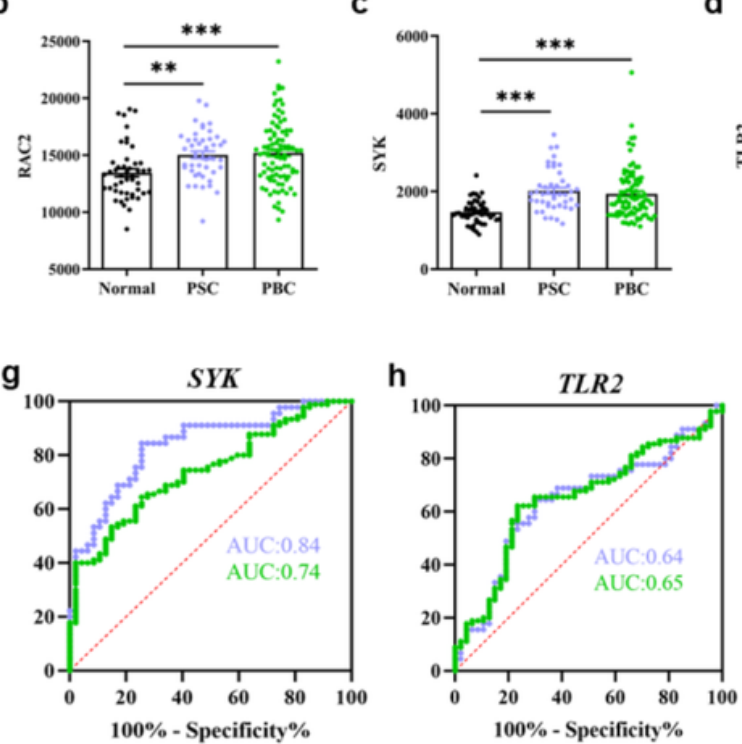

d

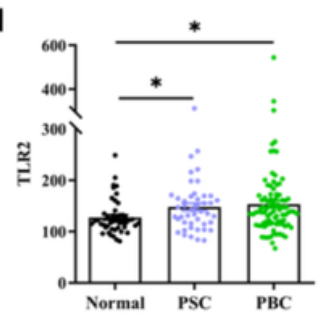

e

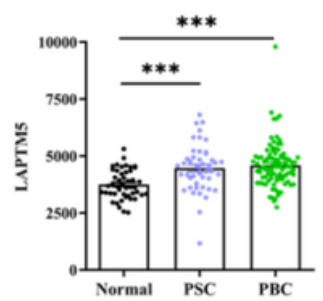

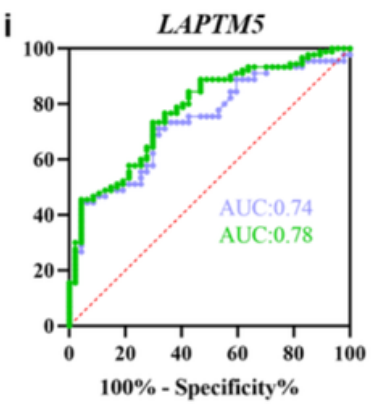

Figure 8

Levels of hub genes in the whole blood of PSC and PBC patients in GSE119600. (a-e) Levels of PTPRC, RAC2, SYK, TLR2, and LAPTM5 in the blood; ( $\mathrm{f}-\mathrm{i})$ ROC curves of RAC2, SYK, TLR2, and LAPTM5. Purple dots represent PSC patients, and green dots represent PBC patients. ${ }^{\star} \mathrm{P}<0.05,{ }^{\star \star} \mathrm{P}<0.01,{ }^{\star \star \star} \mathrm{P}<0.001$.

\section{Supplementary Files}

This is a list of supplementary files associated with this preprint. Click to download.

- Additionalfile1Fig.S1.tif

- Additionalfile2TableS1.docx

- Additionalfile3Fig.S2.pdf

- Additonalfile3Fig.S2legend.docx 